\title{
Global trends and equitable care in endocrine surgery
}

\author{
Ranil Fernando \\ North Colombo Teaching Hospital, Ragama, Sri Lanka
}

Key words: Equitable care; endocrine surgery;

\section{Introduction}

The responsibility of surgeons is to offer the best possible care for the patients. In the era of information technology newer techniques are being introduced every day. Nothing causes dramatic change as the introduction of a revolutionary technology. The newer techniques tend to be expensive and the cost factor creates problems with availability \& equity. What is new may not always be the best. Conflicts between what is available and what is possible arise. The cardinal principle in medicine is that all patients must receive the best possible care irrespective of any other consideration. This is the principle of "Equitable Health Care". In equitable health care, coverage should be universal; be available to most (if not all), should be continuous and should be affordable to individuals and families. Balancing the possibility and availability equation is a difficult task for health systems. If one were to take an objective view of Global Surgical Care, it becomes apparent that, while on the one hand tremendous improvements have been made in technology and it's applications in surgery, most people worldwide though needing a surgery will not receive it. Nor are they likely to see a trained surgeon. This is the number one surgical problem in the world [1].

This stark reality must temper our choices when decisions are made in recommending options in surgery. The ensuing account deals with this in relation to endocrine surgery. The Disease Burden in Endocrine surgery Management of thyroid disease remains the main workload in endocrine surgery (80$90 \%$ ) world over. Goitre remains a problem in most parts of the world especially the low and middle income groups and according to the $\mathrm{WHO} 15.8 \%$ of the world population is likely to be affected by Goitre [2]. There appears to be an increase in the prevalence of thyroid cancer in certain parts of the world $[3,4,5]$. Benign and malignant thyroid disease will be the major burden in endocrine surgery everywhere in the world. Parathyroid disease and adrenal disorders form the rest of the

Correspondence: Ranil Fernando

E-mail: ranilfern@sltnet.lk

Received: 12-11-2017 Accepted: 04-12-2017

(iD) http://orcid.org/0000-0003-4479-1716

DOI: http://doi.org/10.4038/sljs.v35i4.8430

The Sri Lanka Journal of Surgery 2017; 35(4): 1-2 burden while islet cell disorders of the pancreas and other endocrine surgical disorders are rare disease except in specialized referral centres. While being cognizant of the latest developments and trends in thyroid, parathyroid and adrenal surgery, care is needed when recommendations are made in their investigations and treatment, as the small health care budgets in low and middle income countries are likely to cause significant problems with equity of care. The newer methods are costly, and thus there is a potential for reduced availability worldwide.

\section{Global trends and a historical perspective of endocrine surgery}

In the early part of 20th century, pioneering efforts of several surgeons made endocrine surgical procedures, low risk operations.

Thyroidectomy is a very safe surgical procedure with extremely low complication rates quoted by centres with large patient numbers e.g. hypoparathyroidism of less than $2 \%$, injury to recurrent laryngeal nerve $1 \%$, mortality less than $1 \%$. The surgical incisions and operative techniques have changed very little during this time. Currently, utilizing 3-6 $\mathrm{cm}$ collar incisions, modern day surgeons carry out thyroidectomies safely. Standard open thyroidectomy (ST) remains the benchmark in the management of benign and malignant thyroid disease.

The era of endoscopic surgery has come of age now and minimally invasive thyroid surgery is here to stay. Robotics, telesurgery, Lasers, surgical simulators and other advanced technologies are in use in several parts of the world. This raises many new problems for surgeons and decision makers. Is it better to have a PET scanner or to provide access to basic investigations for most? Is endoscopic surgery the best for all types of endocrine surgery? What about cost effectiveness and what about evidence? Is endoscopic surgery justified in parathyroid surgery? Or is it a "market driven exercise" solely aimed at promoting a "minimally invasive parathyroid surgery and an attempt at increasing number of referrals for parathyroid surgery [6]. These questions need answers. The cost effectiveness of endoscopic parathyroidectomy is not established. Less expensive alternatives such as open mini incision parathyroidectomy may be a better alternative [6] 
In terms of evidence, lack of solid evidence in endocrine surgery is one of the main draw backs encountered in many areas of management. At best it is based on experiences of high volume centres with a few randomized trials to support practice. The decision making then becomes difficult and may be tempered and guided by the individual preference of a surgeon. A good example is the use of robotics in thyroid surgery. While robotic thyroidectomy may be called 'minimally invasive', it clearly is not minimally invasive. The approach to the thyroid from a remote access causes a lot of tissue damage not encountered in the open method. In terms of cost, it has been shown that, even in a high income country like USA it is not likely to be available to most people due to its high cost. It may survive as niche operations [7]. In terms of safety, small number of studies, lack of randomization of clinical trials involving this approach leaves many questions unanswered.

Further research, especially in areas of cost benefit analysis and controlled trials with larger numbers, is required to assess the outcome, patient satisfaction, and cost benefit of this surgery $[8,9]$. While newer techniques must be learnt and practiced, the surgeon must be aware of the issues involved and must not lose sight of equitable care based on best available current evidence.

\section{The main issues that need to be addressed in providing equitable care in endocrine surgery}

There are many unanswered questions that need to be considered in providing equitable care in endocrine surgery. They are summarized as follows;

What do we offer our patients?

Do we favour our special interest?

Do we over sell the newer techniques?

Is the industry putting pressure to use certain devices?

Are the decisions based on solid evidence?

How do we attempt to provide equitable care?

Collaborative surgical research may be one method to provide this equity. Every year more than 200 million surgical operations are carried out worldwide [10]. In addition there are other areas that need to be clarified. What do we recommend to the Health System? Each country must decide for itself and the surgeons must recommend to the health care system. Development of guidelines will assist in this. How do we train the postgraduates? Most of them are likely to practice in non-specialized centres. They should able to practice with limited facilities, yet provide good endocrine surgical service. They must undoubtedly be taught the newer techniques. Trainers must be cognizant of steeper learning curve for newer procedures. Open methods need to be learnt by all .What is old is not always bad. Obituary of the open procedures is not due yet. When we are in trouble we always 'open up'. Role of the endoscope in thyroid parathyroid surgery needs irrefutable evidence. In the diseases of the adrenal and endocrine pancreas, endoscopic surgery makes 'sense' as the tissue injury is minimized.

\section{Concluding remarks}

Providing equitable care is the responsibility of all heath care providers and individual surgeons. Technological advances offer alternatives in providing the best medical care for patients. These advances need to be weighed in the balance against their costs. Then only the benefits of these technological advances be accurately calculated against the overall societal cost. The burden that these technological advances place on limited resources is a consideration that surgeons need to bear in mind in making surgical choices. Equitable care in endocrine surgery can then be provided to most within the frame work of emerging global trends if evidence, wisdom and honesty are the guiding principles.

All authors disclose no conflict of interest. The study was conducted in accordance with the ethical standards of the relevant institutional or national ethics committee and the Helsinki Declaration of 1975, as revised in 2000 .

\section{References}

1. Lavy, Christopher, Tavares, Sandra, Roberts Jane Editorial Bulletin of The Royal College of Surgeons of England Volume 95, Number 8, September 2013,pp. 276-276(1)

2. Andersson M, Takkouche B, Egli I, Allen HE, de Benoist B. Iodine Status Worldwide, WHO Global Database on Iodine Deficiency: Department of Nutrition for Health and Development. Geneva: World Health Organization; 2004

3. Vigneri R, Malandrino P, Vigneri P. The changing epidemiology of thyroid cancer: why is incidence increasing? Curr Opin Oncol. 2015 Jan; 27(1):1-7

https://doi.org/10.1097/CCO.0000000000000148

4. Davies L, Welch HG. Increasing incidence of thyroid cancer in the United States, 1973-2002. JAMA 2006; 295:2164-7.

https://doi.org/10.1001/jama.295.18.2164

5. Leenhardt L, Grosclaude P, Cherie-Challine L. Increased incidence of thyroid carcinoma in France: a true epidemic or thyroid nodule management effects? Report from the French Thyroid Cancer Committee. Thyroid 2004; 14:1056-60. https://doi.org/10.1089/thy.2004.14.1056

6. Leigh Delbridge. Minimally Invasive Parathyroidectomy: the Australian Experience Asian Journal of Surgery Vol. 26. No 2; April 2003:76-81

7. Cabot JC, Lee CR, Brunaud L, Kleiman DA, Chung WY, Fahey TJ III, Zarnegar R. Robotic and endoscopic transaxillary thyroidectomies may be cost prohibitive when compared to standard cervical thyroidectomy. Surgery 2012; 152:1016-24. https://doi.org/10.1016/j.surg.2012.08.029

8. Dhaval Patel and Electron Kebebew Pros \& Cons of Robotic Transaxillary Thyroidectomy Thyroid. 2012Oct;22(10):984-985

9. Nicole R. Jackson ,Lu Yao, Ralph P. Tufano, Emad H. Kandil Safety of robotic thyroidectomy approaches: Meta-analysis and systematic review Head \& Neck Volume 36, Issue 1: 137-143, January 2014

10. J. J. Earnshaw and D. Alderson. Sustainable global surgery BJS $2014 ; 101: 1-2$ 